\title{
Alpinia zerumbet (Pers.) B.L. Burtt \& R.M. Sm. (Zingiberaceae): levantamento de publicações nas áreas farmacológica e química para o período de 1987 a 2008
}

CORREA, A.J.C.*; LIMA, C.E.; COSTA, M.C.C.D.

Universidade Católica de Pernambuco - UNICAP, CEP: 50.050-900, Recife-Brasil; *allan_jonathan2003@ hotmail.com

\begin{abstract}
RESUMO: Espécie muito encontrada no nordeste do Brasil, Alpinia zerumbet (Pers.) B.L. Burtt \& R.M. Sm. é conhecida popularmente como colônia. Na medicina popular é utilizada como antihipertensiva, diurética e febrífuga. Alguns estudos com óleos essenciais mostram sua atividade antimicrobiana, hipotensiva e um leve efeito diurético. Visando contribuir para o maior conhecimento da espécie realizou-se uma revisão de publicações envolvendo estudos nas áreas farmacológica e química para o período de 1987 a 2008.
\end{abstract}

Palavras-chave: Alpinia zerumbet, Plantas medicinais, farmacologia

\begin{abstract}
Alpinia zerumbet (Pers.) B.L. Burtt \& R.M. Sm. (Zingiberaceae): survey of publications in pharmacological and chemical areas from 1987 to 2008 . Normally found in the northeast of Brazil, Alpinia zerumbet (Pers.) B.L. Burtt \& R.M. Sm. is commonly known as "colônia". In folk medicine, it has been used as antihypertensive, diuretic and febrifuge. Some studies with essential oils have shown its antimicrobial and hypotensive activities, besides a slight diuretic effect. Aimed at improving the knowledge of such species, a survey of publications involving studies in pharmacological and chemical areas was conducted from 1987 to 2008.
\end{abstract}

Key words: Alpinia zerumbet, medicinal plants, pharmacology

Alpinia zerumbet (Pers.) B.L. Burtt \& R.M. Sm., planta originária da Ásia, pertence à família Zingiberaceae é encontrada na literatura científica com as sinonímias de Alpinia speciosa K. Shum, Costus zerumbetPers., Languas speciosa Small e Zerumbet speciosum J. C. Wendel (Lorenzi \& Souza, 2001).

Trazida para o Jardim Botânico do Rio de Janeiro no século XIX, recebeu o nome de flor-daredenção e bastão-do-imperador (Corrêa, 1975) e atualmente é conhecida também como colônia, pacoseroca, cuité-açu, pacova (Almeida, 1993) e gengibreconcha (Lorenzi \& Souza, 1995).

Trata-se de planta herbácea, perene, que atinge 2 a 3 metros de altura, rizomatosa, com pseudocaule aéreo curto, originado pela sobreposição das bainhas. As folhas são coriáceas, espessas e lanceoladas, curto-pecioladas, verde-luzidias, com bainha aberta, língula desenvolvida e em disposição dística (Almeida, 1993; Lorenzi \& Souza, 2001).

Além de ornamental é muito utilizada entre os populares devido às propriedades medicinais a ela atribuídas. Dentre as propriedades farmacológicas comprovadas para a $A$. zerumbet, destacam-se os efeitos hipotensor e levemente diurético obtidos através do chá das folhas, que foram confirmados pelos estudos de Mendonça et al. (1991) e Laranja et al. (1991, 1992). A atividade antimicrobiana comprovada para óleos essenciais da espécie varia segundo sua composição (Wattiez \& Sternon, 1942). Outras propriedades medicinais, tais como antihistéricas, estomáticas e vermífugas relacionadas às folhas, flores e rizoma foram descritas por Almeida (1993).

Devido ao grande uso popular da espécie e aos riscos inerentes ao uso inadequado, promovemos uma revisão bibliográfica para o período de 1987 a 2008 , visando contribuir para maior conhecimento acerca das propriedades químicas e farmacológicas da espécie, informando sobre as pesquisas científicas realizadas neste período.

Estudos realizados para determinação da composição química de extratos brutos, visando a

Recebido para publicação em 13/11/2008

Aceito para publicação em 01/10/2009

Rev. Bras. PI. Med., Botucatu, v.12, n.1, p.113-119, 2010. 
comprovação de propriedades medicinais para compostos isolados possibilitou a determinação de princípios ativos presentes em extratos brutos e óleos essenciais, bem como a indicação do uso adequado em enfermidades específicas.

Entre as publicações que relacionam compostos químicos isolados da espécie com ações farmacológicas citamos:

Morita et al. (1996), que testaram a ação inibitória de sesquiterpenos e seus derivados isolados de Alpinia japonica e $A$. zerumbet, sobre a histamina ou cloreto de bário, na contração induzida do íleo exposto de porcos da Índia e referiram os sesquiterpenos, $\beta$-eudesmol, nerolide, epóxido humuleno II e $\alpha$-hidroxidihidroagarofurano, como os principais compostos espasmolíticos contidos nos extratos.

Tawata et al. (1996a), que realizaram a síntese e a análise biológica de composto brancocristalino (diidro-5,6-deidrokavaína) isolado das folhas de $A$. zerumbet e a partir da hidrólise deste composto, obtiveram o 4-hidróxi-6-(2-fenil etil)-2H-piran-2-ona, do qual três novos compostos foram sintetizados, entre os quais o dimetil-[6-(2-fenil etil)-2-oxo-2H-piran-4-il] fosforotinado, apresentou grande atividade antifúngica, em torno de $91 \%$ a 100 ppm, frente ao Corticium rolfsii.

No mesmo ano, Tawata et al. (1996b) testaram a volatilidade dos compostos isotimol, timol e eugenol isolados do óleo essencial de A. zerumbet, todos com forte atividade antifúngica contra fitopatogênicos. Concluíram que embora constituintes ativos fossem voláteis no ambiente natural, poderiam ser transformados em fosforotionados não-voláteis pela reação com agentes tiofosfóricos usando como base a tri-tialamina ou $\mathrm{NaOH}_{(\text {aq.) }}$. No estudo, vinte e oito tipos de ésteres tiofosfóricos foram sintetizados e vinte deles mostraram forte atividade antifúngica, promovendo, na concentração de 10 ppm, a inibição de $39,65 \%$ e $56,6 \%$ do crescimento de Pythium sp. e Corticium rolfsii, respectivamente.

Através da utilização de técnicas espectroscópicas, Xu et al. (1996) descobriram dois novos diterpenos do tipo labdano, denominados de Zerumina A e Zerumina $B$, e isolaram das sementes de $A$. zerumbet outros compostos já conhecidos como o 15,16-bisnorlabda-8, 11-dieno-13-ona e coronarin E. Pesquisas mais recentes realizadas com Curcuma amada Roxb., também uma Zingiberaceae, referem a presença da Zerumina B no rizoma desta espécie e atribui a este composto pronunciada atividade citotóxica e antitumoral (Abas et al. 2005).

Mpalantinos et al. (1998) isolaram flavonóides, diidro-5,6-deidrokavaína e 5,6deidrokavaína, do extrato aquoso das folhas de $A$. zerumbet. Referiram que os flavonóides identificados como rutina, campferol-3-O-rutinosídeo, campferol-3O-glucuronido, catequina e epicatequina são substâncias bem conhecidas, que contribuem para a atividade hipotensora, diurética e antiulcerogênica do extrato aquoso da planta e que o diidro-5,6deidrokavaína e 5,6-deidrokavaína atuam como antiulcerogênico e antitrombótico.

Os estudos de Zoghbi et al. (1999), que identificaram os principais componentes do óleo das folhas e flores de $A$. zerumbet, entre os quais o terpineno-4-ol presente tanto nas folhas quanto nas flores, apresenta ação hipotensora. Zoghbi et al. referiram para as folhas além do terpineno-4-ol, que corresponde a $22,7 \%$ dos compostos, o limoneno $(25.1 \%)$ e o $\gamma$-terpineno (17.4\%) e para as flores o 1,8-cineol (23.1\%) e sabineno (14.5\%).

No ano 2000, dois estudos químicos de $A$. zerumbet mereceram destaque: os estudos de Liao et al. (2000) que demonstraram o efeito inibidor de extratos brutos sobre a quantidade de peróxido de lipídios (LPO) formado a partir da hematoporfirina contida na suspensão microssomal do fígado de rato, irradiada com luz visível. Já os estudos de Masuda et al. (2000), que isolou do rizoma de $A$. zerumbet três novos ésteres glucosídeos do ácido ferúlico com função antioxidante.

Após sete anos sem pesquisas na área química para a espécie, Elzaawely et al. (2007a) desenvolveram protocolo para obtenção de óleos essenciais, diidro-5,6-deidrokavaína (DDK) e extratos fenólicos enriquecidos em antioxidante, a partir de folhas frescas e rizoma de $A$. zerumbet. Os principais componentes identificados nos óleos das folhas foram o 1,8-cineol, cânfora e metil cinamato, o primeiro já referido nos estudos de Zoghbi et al. (1999) para extratos das flores. Como principais componentes do óleo essencial do rizoma o autor referiu o DDK e o metil cinamato, sendo DDK, já referido nos estudos realizados por Tawata et al. (1996) e Mpalantinos et al. (1998) para extrato foliar da espécie. Elzaawely et al. (2007a) também referiram que o índice mais elevado de DDK foi encontrado no extrato hexânico dos rizomas frescos. Extratos de acetato de etila das folhas mostraram maior ação antioxidante que extratos do rizoma, o que foi demonstrado pela forte inibição da oxidação de $\beta$-carotenos.

No mesmo ano, testando a composição fenólica de óleos essenciais e extratos de flores e sementes da $A$. zerumbet, Elzaawely et al. (2007b), referiu o 1,8-cineol, a cânfora, o metil cinamato e o borneol, como sendo os principais constituintes dos óleos essenciais das flores, enquanto que os principais componentes dos óleos das sementes foram a alfa-cadinol, o T-muurolol, a alfa-terpinenol, o delta-cadineno e o terpineno-4-ol. A análise da composição fenólica feita, indicou que $o$ ácido $p$ hidroxibenzóico, o ácido ferúlico e ácido siríngico foram 
os fenólicos predominantes no extrato de acetato de etila das flores, enquanto o ácido p-hidroxibenzóico, o ácido siríngico e a vanilina foram os principais fenólicos presentes nas sementes.

Estudos farmacológicos de produtos naturais permitem comprovar a eficácia e a toxicidade de plantas de uso popular e comercializadas. Além disso, certifica os efeitos colaterais, relacionando esses efeitos às doses e a um possível mecanismo de ação em várias espécies de animais de laboratório (Lapa et al., 1999).

Para A. zerumbet a revisão de literatura, feita para o período de 1987 a 2008, mostrou que alguns aspectos farmacológicos da espécie já foram estudados e entre as publicações feitas neste período citamos os estudos de Hsu (1987) que avaliou os efeitos do diidro-5,6-deidrokavaína (DDK) e 5,6deidrokavaína (DK), extraídos do rizoma e das folhas da espécie, sobre cinco tipos de úlceras intestinais agudas induzidas em ratos. Entre os tipos de úlceras estudadas, as úlceras de Shay, induzidas por histamina, úlceras induzidas por aspirinas, dois tipos de úlceras crônicas, induzidas por ácido acético e úlceras termocauterizadas, foram reduzidas por ação de todos os compostos, destacando-se a cura promovida pelo DK e DDK sobre úlceras termocauterizadas.

Avaliando o potencial diurético de Elephantopus scaber e A. zerumbet, Laranja et al. (1991) concluíram que os únicos achados significantes no estudo foram a ação diurética de $A$. zerumbet $(p<0.05)$, e a diminuição da pressão sanguínea diastólica $(p<0,05)$ e sistólica $(p<0,01)$ observada nos 10 voluntários utilizados no estudo (cinco homens e cinco mulheres), com faixa etária entre 20 e 32 anos. Foram administrados a cada um dos voluntários três tipos de chás em doses cinco vezes maiores que o habitual das duas substâncias

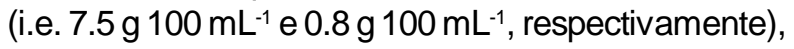
com intervalo de sete dias entre cada dose. Para a comparação entre os efeitos das duas substâncias, foram avaliados os parâmetros sódio plasmático e urinário, potássio, ácido úrico, cálcio, fosfato, uréia e creatinina.

No ano seguinte, Laranja et al. (1992) complementando os estudos acerca da atividade diurética de $A$. zerumbet e $E$. scaber, compararam a ação diurética dessas espécies com a ação diurética de Trandescantia diuretica. Os mesmos procedimentos do estudo anterior foram adotados. Mais uma vez os únicos resultados estatisticamente significativos $(p<0,05)$, foram a redução do volume urinário e diminuição da pressão diastólica e sistólica produzida por $A$. zerumbet. Por não terem sido observadas alterações hidroeletrolíticas e dos parâmetros de função renal, foram excluídos efeitos tubular ou glomerular das espécies testadas.
Estudando os efeitos do óleo essencial de A. zerumbet (EOAZ) no íleo de ratos, Bezerra et al. (2000) verificaram que este óleo na concentração de 0,1-600 $\mu \mathrm{g} \mathrm{mL}^{-1}$ reverte o tônus basal relaxando o íleo. Contrações induzidas por $60 \mathrm{mM}$ de $\mathrm{KCl}$ ou acetilcolina foram inibidas pelo EOAZ com valores similares a $\mathrm{IC}_{50}\left(\sim 44 \mathrm{e} 48 \mu \mathrm{g} \mathrm{mL}^{-1}\right.$, respectivamente). Estes resultados mostraram que o EOAZ possui tanto ação relaxante como antiespasmódica sobre o íleo. Esta ação pode ser explicada pela presença de sesquiterpenos e derivados no óleo de $A$. zerumbet, que nos estudos de Morita (1996) é apontado como grupo químico de principal atividade espasmolítica.

Analisando os terpenos contidos nos óleos essenciais de $A$. zerumbet, Moreira et al. (2001) estudaram os efeitos sobre o potencial de ação dos nervos ciáticos de ratos. Verificaram que os terpenos induziram o bloqueio dose-dependente do potencial de ação nas doses de $300 \mu \mathrm{M}$ e $600 \mu \mathrm{M}$, com as quais foram alcançados picos de amplitude e velocidade de condução do potencial de ação os quais foram significativamente reduzidos ao término de 180 minutos de exposição do nervo ao fármaco.

Estudando a atividade antioxidante de espécies cultivadas em Okinawa (Japão) e utilizadas como comestíveis e medicinais, Masuda et al. (2002) comprovaram forte atividade redutora do radical 1.1difenil-2-picrilhidrazil (DPPH) promovida pelos extratos do rizoma de $A$. zerumbet e potente atividade inibitória da lipoperoxidação promovida por extratos de frutos e rizomas. Concluíram uma potente capacidade antioxidativa para a espécie, já referida por eles em estudos quando isolaram antioxidantes do rizoma de A zerumbet.

Analisando o efeito do óleo essencial de $A$. zerumbet sobre o potencial de ação composto (CAP) no nervo ciático de ratos, Leal-Cardoso et al. (2004) verificaram que o óleo essencial da espécie induziu o bloqueio dose dependente do CAP. A dose de $60 \mu \mathrm{g}$ $\mathrm{mL}^{-1}$ do óleo essencial não induziu nenhum efeito sobre o pico de amplitude e nem sobre a velocidade de condução do CAP, porém na dose de $100 \mu \mathrm{g} \mathrm{mL}$ ${ }^{1}$ reduziu significativamente a velocidade de condução. Doses de 300, 600 e $2000 \mu \mathrm{g} \mathrm{mL}^{-1}$ de óleo essencial reduziram significativamente o pico de amplitude do nervo ciático, num período de 180 minutos em ambos os casos.

No mesmo ano, verificando os efeitos antinociceptivos do óleo essencial de $A$. zerumbet administrado por via oral em camundongos Swiss masculinos (20-25 g cada um), Araújo et al. (2005) observaram que nas contrações induzidas pelo ácido acético, o óleo essencial foi efetivo em todas as doses testadas $\left(30,100\right.$ e $300 \mathrm{mg} \mathrm{kg}^{-1} ; \mathrm{n}=10, \mathrm{n}=13$ e $\mathrm{n}=$ 15 , respectivamente). Nos testes da placa-quente, A. zerumbet aumentou significativamente a latência 
nas doses de 100 e $300 \mathrm{mg} \mathrm{kg}^{-1}$ de peso, porém não na dose de $30 \mathrm{mg} \mathrm{kg}^{-1}$ nas observações cronometradas por 180 minutos ( $\mathrm{n}=10$ para cada dose). Os autores concluíram que a administração oral da $A$. zerumbet promoveu efeito antinociceptivo dose-dependente, com mecanismo de ação que envolve, provavelmente, a participação dos receptores opióides.

A fim de elucidar a ação antihipertensiva da A. zerumbet, cinco estudos referentes à atividade hipotensora da espécie foram publicados no período correspondente a esta revisão, dentre eles os estudos feitos por Lordelo et al. (2000) que determinaram a atividade antihipertensiva e diurética, bem como, a toxicologia clínica de $A$. zerumbet, administrando extratos da espécie, sob a forma de folhas secas pulverizadas e encapsuladas, em pacientes com diagnóstico de hipertensão arterial essencial, estágio I (leve) ou II (moderada). Os pacientes avaliados não apresentaram modificações do volume ou da freqüência urinária, bem como alterações nos exames laboratoriais. Após os estudos, os autores concluíram que cápsulas de $A$. zerumbet foram bem toleradas, seguras e eficazes no controle da hipertensão essencial leve ou moderada, em aproximadamente $70 \%$ dos pacientes. Segundo o que Mpalantinos (1998) referiu nos estudos, essa atividade se explica pela presença de flavonóides no extrato foliar de $A$. zerumbet que contribuem para a atividade antihipertensiva e diurética.

Investigando o efeito do extrato hidroalcoólico de $A$. zerumbet (EHAZ), Emiliano (2002) isolou o leito vascular mesentérico (LVM) de ratos Wistar machos (250-350 g). Os estudos demonstraram que o EHAZ promoveu potente efeito vasodilatador no LVM, e que este efeito parece ser endotélio-dependente. Os resultados permitiram a autora concluir que o efeito antihipertensivo do EHAZ, observado na medicina popular, é decorrente de vasodilatação com consequente diminuição da resistência vascular periférica.

Lahlou et al. (2002), estudando os efeitos cardiovasculares no tratamento intravenoso com óleos essenciais de $A$. zerumbet em ratos conscientes e anestesiados por pentobarbitona, observaram que 0 tratamento utilizado induzia hipotensão imediata e significante, que pode ser parcialmente atribuída a ação do terpineno-4-ol. Com os estudos, os autores confirmaram os resultados referidos por Zoghbi et al. (1999), que já haviam referido a ação antihipertensiva para o terpineno-4-ol presente tanto nas folhas quanto no rizoma de $A$. zerumbet.

No ano seguinte, Lahlou et al. (2003) continuaram os estudos sobre a ação cardiovascular de $A$. zerumbet, desta vez analisando as respostas hipotensivas promovidas pelo tratamento intravenoso com óleo essencial da espécie em ratos. Os resultados mostraram que o tratamento intravenoso com óleo essencial de $A$. zerumbet ou com terpineno4-ol (Trp-4-ol) dose dependente, decresceram a pressão sanguínea nos ratos hipertensos e esta ação foi realçada quando comparada com controles uninefrectomizados. Com estes dados eles reafirmaram a hipótese que os efeitos hipotensivos da $A$. zerumbet estão atribuídos parcialmente às ações do Trp-4-ol.

Moura et al. (2005) realizaram experimentos com extratos hidroalcoólicos das folhas de $A$. zerumbet, para verificar o efeito vasodilatador sobre a camada vascular mesentérica e o efeito antihipertensivo em ratos com hipertensão induzida por cloreto de sódio (DOCA). Em vasodilatação, précontrolada com norepinefrina, a $A$. zerumbet induziu uma vasodilatação endotélica. Em vasos précontraídos pela norepinefrina, o efeito vasodilatador da espécie não foi alterado pela 4-aminopiridina, glibenclamida ou pela apamina plus caribdotoxina. Os autores verificaram que o efeito vasodilatador $e$ antihipertensivo da espécie foram comprovados pela redução significativa na pressão arterial sistólica, média e diastólica nos ratos hipertensos.

Sete publicações referentes à atividade antimicrobiana da $A$. zerumbet foram encontradas no período correspondente a revisão, indicando que a ação antimicrobiana da espécie, embora não seja a principal motivação para o seu uso popular, uma vez que é usada principalmente como antihipertensiva, vem sendo investigada por diferentes pesquisadores.

Lobato et al. (1989) avaliaram a atividade antimicrobiana de óleos essenciais de quatorze plantas usadas na região amazônica em banhos aromáticos e como desinfetantes de roupas e armários. Os óleos de $A$. zerumbet foram ativos frente a oito das nove bactérias testadas, dentre elas Staphylococus aureus, Proteus mirabilis, Proteus vulgaris, Escherichia coli, Edwardsiella tarda, Klebsiella pneumoniae, Enterobacter aerogenes e Salmonella sp., foram sensíveis aos óleos essenciais com halos de inibição variando de 7 a $14 \mathrm{~mm}$.

Yu et al. (1993) realizaram estudos comparativos da bioatividade de cinco espécies medicinais, entre elas $A$. zerumbet, e os resultados obtidos no estudo mostraram que os óleos voláteis de $A$. aurantiacum e de $A$. zerumbet foram ativos frente a bactérias Gram-negativas.

No ano seguinte, extratos de dez plantas medicinais foram testados por Sá et al. (1994), frente a bactérias causadoras de conjuntivite como Streptococcus aureus, Pseudomonas aeruginosa, Bacillus subtilis, Streptococcus pneumoniae, Streptococcus pyogenes, Neisseria gonorrhoeae. Os extratos de $A$. zerumbet, bem como os de Laurus nobilis, Cymbopogon citratus, Pimpinella anisum e Eugenia uniflora apresentaram atividade antimicrobiana produzindo halos de inibição acima 
de 10 mm de diâmetro.

Cinqüenta extratos etanólicos de plantas medicinais de uso popular no Taiwan foram testados por Wang \& Huang (2005) sobre o Helicobacter pylori. A. zerumbet figurou entre as espécies que apresentaram atividade antimicrobiana frente ao microrganismo testado com concentrações mínimas inibitórias do extrato etanólico variando entre 0,64 a $10,24 \mathrm{mg} \mathrm{mL}^{-1}$.

No mesmo ano, Voravuthikunchai et al. (2005) avaliaram atividade antimicrobiana para doze plantas medicinais da Tailândia que são utilizadas na automedicação por pacientes de HIV/AIDS do país. Trinta e nove extratos (clorofórmicos, metanólicos e aquosos) das plantas, entre as quais $A$. zerumbet, foram testados frente aos microrganismos $S$. aureus, Staphylococcus aureus metacilina resistente (MRSA), Streptococcus mutans e Salmonella typhi, comumente associados com infecções em aidéticos. As bactérias Gram-positivas apresentaram-se suscetíveis frente ao extrato clorofórmico de sete espécies, inclusive a $A$. zerumbet.

Procurando investigar a atividade antimicrobiana e antioxidante, assim como quantificar o diidro-5,6-deidrokavaína (DDK), o nível de fenólicos totais e os óleos essenciais de $A$. zerumbet,em plantas tratadas e não tratadas com o sulfato de cobre por 24 horas, Elzaawely et al. (2006) demonstraram que plantas tratadas com sulfato de cobre apresentaram maior nível de fenólicos totais, maior ação antioxidante e antibacteriana frente ao Bacillus cereus do que aquelas não tratadas. O conteúdo de DDK, vanilina e de ácido cinâmico foram significativamente maiores nos extratos clorofórmicos e acetato de etila das plantas expostas ao cobre. Concluíram que a aplicação foliar do sulfato de cobre pode regular os compostos antioxidantes em plantas da $A$. zerumbet, influenciando sua atividade antioxidante e antibacteriana.

Os extratos hidroalcoólico, hexânico e clorofórmico da $A$. zerumbet foram testados frente a nove microorganismos, como $S$. aureus, $B$. subtilis, Enterococcus faecalis, Micrococcus luteus, E. coli, $P$. aeruginosa, Serratia marcescens, Mycobacterium smegmatis e Candida albicans por Costa et al. (2007a), com bons resultados do extrato clorofórmico do rizoma frente a $E$. faecalis e no extrato hexânico de folhas frente a $C$. albicans.

No ano seguinte, continuando os estudos de atividade antimicrobiana de $A$. zerumbet, Costa et al. (2008) testaram extratos metanólico e acetônico frente aos mesmos microrganismos utilizados no estudo anterior, com bons resultados para o extrato acetônico do rizoma frente à $S$. aureus, $B$. subtilis, $E$. faecalis, M. luteus, para os quais foram obtidos halos superiores a $20 \mathrm{~mm}$. A Concentração Mínima Inibitória do extrato acetônico do rizoma frente a $S$. aureus, $B$. subtilis e $M$. luteus foi equivalente a 125 $\mu \mathrm{g} \mathrm{mL}^{-1}$ e frente a $E$. faecalis foi igual a para e 500 $\mu g \mathrm{~mL}^{-1}$.

Apenas duas publicações foram encontradas sobre a atividade citotóxica de $A$. zerumbet no período correspondente a esta revisão.

Costa et al. (2007b) testaram a atividade citotóxica dos extratos clorofórmicos, hexânicos e hidroalcoólicos em $A$. zerumbet, Chenopodium ambrosioides e Acmella oleracea sobre quatro linhagens celulares cancerígenas (HEp-2, NCl-H292, $\mathrm{KB}$ e HeLa). Os resultados mostraram ausência de citotoxicidade significativa para todos os extratos testados frente a estas linhagens celulares.

No ano seguinte, dando continuidade aos estudos da atividade citotóxica de $A$. zerumbet, Corrêa \& Costa (2008) testaram os extratos acetônico e metanólico da espéice, sobre células $\mathrm{HEp}-2, \mathrm{NCl}-$ H292 e KB. Os resultados também mostraram ausência de citotoxicidade significativa para os extratos testados.

Pode se concluir que as publicações para área citotóxica de $A$. zerumbet de fato não se fazem presentes em bancos de dados de pesquisa para periódicos, mostrando com isso, o quanto essa espécie ainda apresenta aspectos tóxicos importantes a serem descobertos, mas fortalece também as principais propriedades medicinais como atividade antihipertensiva e diurética, que tiveram bastantes publicações, o que nos faz entender 0 quanto são importantes as revisões bibliográficas, pois além de dar embasamento teórico e metodológico, mostram também áreas de conhecimento que ainda não foram exploradas por outros pesquisadores, facilitando assim, a escolha destas áreas para os estudos futuros.

\section{REFERÊNCIA}

ABAS, F. et al. A labdane diterpene glucoside from the rhizomes of Curcuma mangga. Journal of Natural Products, v.7, n.68, p.1090-3, 2005.

ALMEIDA, E.R. Plantas medicinais brasileiras: conhecimentos populares e científicos. São Paulo: Hemus Editora, 1993. 341p.

ARAÚJO, P.F. et al. Antinociceptive effects of the essential oil of Alpinia zerumbet on mice. Phytomedicine, v.12, n.6-7, p.482-6, 2005

BEZERRA, M.A.C. et al. Myorelaxant and antispasmodic effects of the essential oil of Alpinia speciosa on rat ileum. Phytotherapy Research, v.14, n.7, p.549-51. 2000. Disponível em: <http://www3.interscience.wiley.com/ journal/73505647/ abstract>. Acesso em: 15 ago. 2006. CORRÊA, P. Dicionário das plantas úteis do Brasil. Rio de Janeiro: Ministério da Agricultura e IBDF, 1975. v.6, $345 p$.

CORRÊA, A.J.C.; COSTA, M.C.C.D. Estudo da atividade antimicroiana e citotoxica de extratos brutos de Alpinia

Rev. Bras. Pl. Med., Botucatu, v.12, n.1, p.113-119, 2010. 
speciosa K. Shum. In. JORNADA DE INICIAÇÃO CIENTÍFICA, 10., 2008, Recife. Resumos... Recife: Universidade Católica de Pernambuco, 2008. CD-ROM, p.25.

COSTA, M.C.C.D. et al. Avaliação da atividade antimicrobiana de extratos brutos de alpinia speciosa $\mathrm{k}$. Schum (zingiberaceae). In: REUNIÃO NORDESTINA DE BOTÂNICA, 30., 2007, Crato. Resumos ... Crato: Universidade Regional de Cariri-URCA, 2007a. CD-ROM, p.31.

COSTA, M.C.C.D. et al. Avaliação da atividade citotóxica de Acmella oleracea (L) R.K. Jansen, Alpinia speciosa K. Shum e Chenopodium ambrosioides L. In: SIMPÓSIO DE PLANTAS MEDICINAIS DO VALE DO SÃO FRANCISCO, 1., 2007, Petrolina. Anais... Petrolina: Universidade do Vale do São Francisco-UNIVASF, 2007b. CD ROM, p.18.

COSTA, M.C.C.D. et al. Determinação da concentração mínima inibitória para extratos brutos de Alpinia speciosa K. Shum com boa atividade antimicrobiana. In: SIMPÓSIO DE PLANTAS MEDICINAIS DO BRASIL, 20., 2008, São Paulo. Anais... São Paulo: Eventus, 2008. CD ROM, p.903. COSTA, M.C.C.D.; CORRÊA, A.J.C. Estudo da atividade antimicroiana e citotoxica de extratos brutos de Alpinia speciosa K. Shum. In. JORNADA DE INICIAÇÃO CIENTÍFICA, 10., 2008, Recife. Anais... Recife: Universidade Católica de Pernambuco, 2008. CD ROM, p.25.

ELZAAWELY, A.A.; XUAN, T.D.; TAWATA, S. Changes in essential oil, kava pyrones and total phenolics of Alpinia zerumbet (pers.) B.I. Burtt. \& R.M. Sm. leaves exposed to copper sulphate. Environmental and Experimental Botany, v.59, n.3, p.347-53, 2006.

ELZAAWELY, A.A. Antioxidant activity and contents of essential oil and phenolic compounds in flowers and seeds of Alpinia zerumbet (Pers.) BL Burtt \& RM Sm. Food Chemistry, v.104, n.4, p.1648-53, $2007 \mathrm{a}$.

ELZAAWELY, A.A. Essential oils, kava pyrones and phenolic compounds from leaves and rhizomes of A/pinia zerumbet (pers.) B.I. Burtt \& R.M. Sm. And their antioxidant activity. Food Chemistry, v.103, n.2, p.486-94, 2007b. EMILIANO, A.F. Efeito vasodilatador do extrato hidroalcóolico da Alpinia zerumbet (Pers.) Burtt e Smith no leito vascular mesentérico. 2002, 74p. Dissertação (Mestrado - Área de Fisiopatologia) - Departamento de Fisiopatologia Clinica e Experimental, Universidade Estadual do Rio de Janeiro, Rio de Janeiro.

HSU, S. Effects of the constituents of Alpinia speciosa rhizome on experimental ulceras. Journal of the Formosan Medical Association, v.86, n.1, p.58-64. 1987. LAHLOU, S. et al. Cardiovascular effects of the essential oil of Alpinia zerumbet leaves and its main constituent, Terpinen-4-ol, in rats: role of the autonomic nervous system. Planta Medica, v.68, p.1097-102, 2002.

LAHLOU, S. et al. Antihypertensive effects of the essential oil of Alpinia zerumbet and its main constituent, terpinen4-ol, in DOCA-salt hypertensive conscious rats Fundamental \& Clinical Pharmacology, v.17, n.3, p.32330, 2003.

LAPA, A.J. et al. Farmacologia e toxicologia de produtos naturais. In: SIMÕES, C.M.O. et al. (Org.). Farmacognosia: da planta ao medicamento. Porto Alegre: Editora da Universidade do Rio Grande do Sul, 1999. p.181-95. LARANJA, S.M.R.; BERGAMASCHI, C.M.; SCHOR, N.
Evaluation of acute administration of natural products with potential diuretic effects, in humans. Memórias do Instituto Oswaldo Cruz, v.86, p.237-40, 1991.

LARANJA, S.M.R.; BERGAMASCHI, C.M.; SCHOR, N. Avaliação de três plantas com potencial diurético. Revista Associação Médica Brasileira, v.38, n.1, p.13-6, 1992. LEAL-CARDOSO, J.H. et al. Effects of essential oil of Alpinia zerumbet on the compound action potential of the rat sciatic nerve. Phytomedicine, v.11, n.6, p.549-53, 2004.

LIAO, M.C. et al. Inhibitory effects of Alpinia speciosa K. SCHUM. On the porphyrin photooxiative reaction. The Journal of Dermatology, v.27, n.5, p.312-7, 2000.

LOBATO, A.M. et al. Antimicrobial activity of essential oils from Amazônia. Acta Amazônica, v.19, n.1, p.355-65, 1989. LORDELO, I.G.S. et al. Avaliação clínica e toxicológica da Alpinia zerumbet (Pers.) Brustt \& Smith em pacientes com hipertensão arterial estágio I (leve) ou estágio II (moderada). In: SIMPÓSIO DE PLANTAS MEDICINAIS DO BRASIL, 16., 2000, Recife. Resumos... Recife: Sociedade Brasileira de Plantas Medicinais, 2000.

LORENZI, H.; SOUZA, H.M. Plantas ornamentais no Brasil: arbustivas, herbáceas e trepadeiras. São Paulo: Plantarum, 1995. 720p.

LORENZI, H.; SOUZA, H.M. Plantas ornamentais no Brasil: arbustivas, herbáceas e trepadeiras. 3.ed. São Paulo: Instituto Plantarum, 2001. 1067p.

MASUDA, T. et al. Isolation and structure determination of new antioxidative ferulic acid glucoside esters from rhizome of Alpinia speciosa, a Zingiberaceae plant used in Okinawan food culture. Jornal of Agriculture and Food Chemistry, v.48, n.5, p.1479-84, 2000.

MASUDA, T. et al. Antioxidant-related activities of ethanol extracts from edible and medicinal plants cultivated in Okinawa, Japan. Journal of the Japanese Society for Food Science and Technology-Nippon Shokuhin Kagaku Kogaku Kaishi, v.49, n.10, p.652-61, 2002.

MENDONÇA, V.L.M. et al. Pharmacological and toxicological evaluation of Alpinia speciosa. Memórias do Instituto Oswaldo Cruz, v.86, p.93-7, 1991.

MOREIRA, M.R. et al. Effects of terpineol on the compound action potential of the rat sciatic nerve. Brazilian Journal Of Medical And Biological Research, v.34, n.10, p.133740, 2001.

MORITA, M. et al. Structures and spasmolytic activities of derivatives from sesquiterpenes of Alpinia speciosa and Alpinia japonica. Chemical \& Pharmaceutical Bulletin, v.44, n.8, p.1603-6, 1996.

MOURA, R.S. et al. Antihypertensive and endotheliumdependent vasodilator effects of Alpinia zerumbet, a medicinal plant. Journal Cardiovascular Pharmacology, v.46, n.3, p.288-94, 2005.

MPALANTINOS, M.A. et al. Biologically active flavonoids and kava pyrones from the aqueous extract of Alpinia zerumbet. Phytotherapy Research, v.12, n.6, p.442-4, 1998.

SÁ, L.D. et al. Estudo sobre atividade antimicrobiana de extratos vegetais sobre bactérias causadoras de conjuntivite. Revista Brasileira de Ciências da Saúde, v.13, n.1, p.13-7, 1994.

TAWATA, S. et al. Syntheses and biological activities of dihydro-5,6-dehydrokawain derivatives. Bioscience, Biotechnology and Biochemistry, v.60, n.10, p.1643-5, 
1996a.

TAWATA, S. et al. Synthesis and fungicidal activity of new thiophosphorylated monoterpenoids and related compounds. Journal of Pesticide Science, v.21, n.2, p.141-6 1996b.

VORAVUTHIKUNCHAI, S.P.; PHONGPAICHIT, S.; SUBHADHIRASAKUL, S. Evaluation of antibacterial activities of medicinal plants widely used among AIDS patients in Thailand. Pharmaceutical Biology, v.43, n.8, p.701-6, 2005.

WANG, Y.C.; HUANG, T.L. Screening of anti-Helicobacter pylori herbs deriving from Taiwanese folk medicinal plants. FEMS Immunology and Medical Microbiology, v.43, n.2, p.295-300, 2005.

WATTIEZ, N.; STERNON, F. Élements de chemie végétale. 2.ed. Paris: Masson et Cie, 1942. 844p.

XU, H.; DONG, H.; SIM, K. Labdane diterpenes from Alpinia zerumbet. Phytochemistry, v.42, n.1, p.149-51, 1996.

$\mathrm{YU}$, B. et al. Comparative studies on bioactivities of the resources plants of Fructus amomi. Journal of Plant Resources and Environment, v.2, n.3, p.18-23, 1993.

ZOGHBI, M.G.B. et al. Volatile constituents from leaves and flowers of Alpinia speciosa K. Schum. and $A$. pupurata (Viell.) Schum. Flavour and Fragrance Journal, v.14, n.6, p.411-4, 1999. 